\title{
Trans Complementation of Replication-defective Omsk Hemorrhagic Fever Virus for Antiviral Study
}

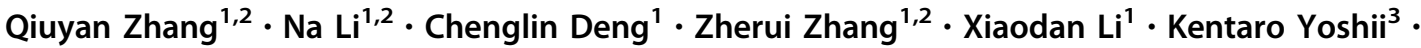 \\ Hanqing $\mathrm{Ye}^{1} \cdot$ Bo Zhang $^{1}$ (i)
}

Received: 16 January 2019/Accepted: 28 February 2019/Published online: 4 April 2019

(c) Wuhan Institute of Virology, CAS 2019

\begin{abstract}
Omsk hemorrhagic fever virus (OHFV) is a tick-borne flavivirus classified as a biosafety level-4 (BSL4) pathogen. Studies of OHFV are restricted to be conducted within BSL4 laboratories. Currently, no commercial vaccines or antiviral drugs are available against OHFV infection. In this study, we recovered a replication-deficient OHFV with an NS1 deletion (OHFV$\Delta$ NS1) and reporter virus replacing NS1 with the Gaussia luciferase (Gluc) (OHFV- $\Delta$ NS1-Gluc). Both the defective OHFV$\Delta \mathrm{NS} 1$ and OHFV- $\Delta \mathrm{NS} 1-$ Gluc virus could only replicate efficiently in the BHK21 cell line expressing NS1 (BHK21 $1_{\mathrm{NS} 1}$ ) but not in naïve BHK21 cells. The Gluc reporter gene of OHFV- $\triangle$ NS1-Gluc virus was maintained stably after serial passaging of BHK21 $1_{\mathrm{NS} 1}$ cells and was used to surrogate the replication of OHFV. Using NITD008, OHFV- $\Delta$ NS1-Gluc virus was validated for antiviral screening, and high-throughput screening parameters were optimized in a 96-well plate format with a calculated $\mathrm{Z}^{\prime}$ value above 0.5 . The OHFV- $\Delta \mathrm{NS} 1-$ Gluc reporter virus is a powerful tool for antiviral screening as well as viral replication and pathogenesis studies in BSL2 laboratories.
\end{abstract}

Keywords Omsk hemorrhagic fever virus (OHFV) - Trans complementation · NS1 - Gaussia luciferase (Gluc) · Antiviral screening

\section{Introduction}

Omsk hemorrhagic fever (OHF) is caused by Omsk hemorrhagic fever virus (OHFV), which belongs to the tickborne encephalitis (TBE) serocomplex, genus Flavivirus, family Flaviviridae. The Flavivirus genus includes many other important pathogens such as dengue virus (DENV), zika virus (ZIKV), West Nile virus (WNV), Japanese encephalitis virus (JEV), yellow fever virus (YFV), and tick-borne encephalitis virus (TBEV). The clinical

Bo Zhang

zhangbo@wh.iov.cn

1 Key Laboratory of Special Pathogens and Biosafety, Center for Emerging Infectious Diseases, Wuhan Institute of Virology, Chinese Academy of Sciences, Wuhan 430071, China

2 University of Chinese Academy of Sciences, Beijing 100049, China

3 Laboratory of Public Health, Graduate School of Veterinary Medicine, Hokkaido University, Sapporo 060-0818, Japan symptoms of OHF include fever, headache, cough, myalgia, hemorrhage, and sometimes meningeal signs with neurological system damage, and the mortality rate ranges from $0.5 \%$ to $3 \%$ (Charrel et al. 2004; Ruzek et al. 2010). Since its first isolation in 1947 from a human presenting with hemorrhagic fever in Omsk area (Dobler 2010; Lani et al. 2014), OHFV had become prevalent in Omsk, Novosibirsk, Tyumen, and Kurgan in some regions of western Siberia in Russia (Charrel et al. 2004; Ruzek et al. 2010).

OHFV is transmitted by the bites of the meadow tick Dermacentor reticulatus (Charrel et al. 2004; Ruzek et al. 2010) and contact with infected vole (Microtus gregalis) and muskrats (Ondatra zibethicus). It had been reported that the introduction of muskrats, which are sensitive to OHFV, caused the virus to spread widely (Ruzek et al. 2010). OHF is an example of a human disease that has emerged because of human-mediated disturbances to an ecological niche. The widespread presence of ticks enabled the global spread of the virus. Additionally, OHFV can be transmitted by aerosol and contaminated water which 
played important roles in the spread of the virus to humans and wider propagation of this virus (Dobler 2010; Lani et al. 2014). Although the morbidity and mortality of infection are low, many cases of human infection may be undiagnosed because of the lack of effective diagnostic methods. Because of its numerous transmission routes, OHFV may spread worldwide and become prevalent, as observed for TBEV. Although a recombinant TBEV vaccine may cross-protect against OHFV (Orlinger et al. 2011), no licensed vaccine or compound is available against OHFV. Therefore, additional antiviral screening, vaccine development, and pathogenesis studies of OHFV are needed.

OHFV is classified as a biosafety level-4 (BSL4) pathogen, limiting further progress in OHFV research because of insufficient BSL4 containment facilities. To overcome this limitation, researchers have developed numerous alternatives for studying BSL3 or BSL4 viruses in BSL2 laboratories. A noncytopathic replicon system and virus-like particle (VLP) system with reporter genes have been constructed for several BSL3 viruses such as chikungunya virus (Pohjala et al. 2011), TBEV (Hayasaka et al. 2004; Yoshii et al. 2009), and WNV (Shi et al. 2002; Scholle et al. 2004; Pierson et al. 2006), as well as for BSL4 viruses such as Ebola virus (Wang et al. 2016) for screening inhibitors, neutralization test and vaccine development. Viral replicons do not contain structural proteins and only perform transcription and replication functions. VLPs generated by the packaging of replicon RNA with structural proteins in trans are efficient tools for substituting wild-type virus to monitor the entire viral life cycle.

Additionally, replication-defective viruses are typically produced by exogenously supplied proteins or other factors to complement the deficient genome with gene deletions or mutations. Defective viruses reproduce only in complementary cells and function similarly to the wild-type virus. This replication-defective virus system has been generated for the Ebola virus with VP30 gene deletion (Ebola $\Delta$ VP30-neo virus) for propagation in the Vero cell line stably expressing VP30 protein (Halfmann et al. 2008). The viruses resemble wildtype virus in their life cycle, but can be handled in a non-BSL4 laboratory. Moreover, a similar strategy based on the replication-defective system was used for influenza A viruses (BSL3) (Si et al. 2016). Because of the replication defects of the genome itself, these defective viruses cannot replicate in conventional cells but only propagate in complementary cells; thus, replication-defective viruses are safe for use in BSL2 laboratories for studying high biosecurity level viruses.

OHFV genome is a single-stranded, positive-sense RNA approximately $11 \mathrm{~kb}$ in length and consists of a large open reading frame flanked by $5^{\prime}$ and $3^{\prime}$ untranslated regions. The genome is translated into a polyprotein that is processed into three structural proteins $(\mathrm{C}, \mathrm{prM}$, and $\mathrm{E})$ and seven nonstructural proteins (NS1, NS2A, NS2B, NS3, NS4A, NS4B, and NS5). The structural proteins play an important role in virion entry, fusion, and assembly. The nonstructural proteins function in replication complex formation and viral genome replication. Among them, NS1 is a multifunctional glycoprotein secreted into the extracellular space and modulates early viral RNA replication and immune evasive functions (Lindenbach and Rice 1997; Youn et al. 2012; Zhang et al. 2017b). Deletion of NS1 from the viral genome abrogates replication. However, it had been reported that NS1-deleted YFV and WNV can be complemented in trans by exogenous expression of NS1 and packaged into a replication-defective virus (Lindenbach and Rice 1997; Khromykh et al. 2000; Zhang et al. 2017a).

Few antiviral screening and pathogenicity studies have evaluated alternatives for replacing OHF live virus without the requirement for BSL4 facilities. Only OHFV VLPs packaging replicons with structural proteins have been established for neutralization testing of the strain Guriev (Yoshii and Holbrook 2009), while inhibitor screening is still performed using authentic viruses (Lo et al. 2016). Thus, it is necessary to develop a novel, safe, and stable system for antiviral screening against OHFV in BSL2 laboratories.

In this study, replication-deficient OHFV- $\triangle \mathrm{NS} 1$ and OHFV- $\Delta$ NS1-Gluc viruses were produced by complementation with a deficient genome using the BHK $21_{\mathrm{NS} 1}$ cell line expressing NS1 in trans. Both defective viruses replicated only efficiently in the BHK21 $1_{\mathrm{NS} 1}$ cell line without reversion to the wild-type virus after serial passaging, and could not replicate in naïve BHK21 cells, demonstrating its safety. Luciferase activity can be used to quantify the viral replication of OHFV- $\Delta$ NS1-Gluc. Using a known inhibitor of flaviviruses, NITD008, we found that the luciferase activities derived from OHFV- $\Delta$ NS1-Glucinfected BHK2 $1_{\mathrm{NS} 1}$ cells were inhibited in a concentrationdependent manner, demonstrating that OHFV- $\Delta$ NS1-Gluc can be used for antiviral screening. A high-throughput screening (HTS) assay using the OHFV- $\Delta$ NS1-Gluc on BHK21 $1_{\mathrm{NS} 1}$ cell line was optimized, showing a $Z^{\prime}$ value above 0.5. In general, a stable and safe replication-deficient OHFV system was developed for use in antiviral studies in a BSL2 laboratory.

\section{Materials and Methods}

\section{Cells, Antibodies, and Reagents}

BHK21 and 293T cells were cultured in Dulbecco's modified Eagle's medium supplemented with $10 \%$ fetal bovine 
serum, $100 \mathrm{U} / \mathrm{mL}$ of penicillin, and $100 \mu \mathrm{g} / \mathrm{mL}$ of streptomycin at $37{ }^{\circ} \mathrm{C}$ with $5 \% \quad \mathrm{CO}_{2}$. The $\mathrm{BHK} 21_{\mathrm{NS} 1}$ cell line stably expressing OHFV-NS1 was grown in complete medium containing $0.8 \mu \mathrm{g} / \mathrm{mL}$ puromycin (Sigma, St. Louis, MO, USA). The antibody anti-HA tag was purchased from Cell Signaling Technology (Danvers, MA, USA) and used for Western blotting and immunofluorescent assays (IFA) to detect the expression of OHFV-NS1. The antibody against OHFV-NS3 was obtained by immunizing mice with NS3 protein and used for IFA. The secondary antibodies, horseradish peroxidase-conjugated goat anti-mouse antibody and fluorescein isothiocyanate-conjugated goat anti-mouse IgG (purchased from ProteinTech Group, Rocky Hill, NJ, USA) were used for Western blotting and IFA, respectively. NITD008 was synthesized as reported previously (Yin et al. 2009).

\section{Plasmid Construction}

OHFV full-length infectious clone (OHF-IC) was kindly provided by Professor Yoshii et al. (2011). According to a previous strategy, the truncated NS1 fragment with a deletion of amino acids (aa) 4-298 was generated by fusion PCR using OHF-IC as a template (Khromykh et al. 2000; Zhang et al. 2017a). The engineered fragment was then inserted into OHF-IC between the restriction sites BsrG I and $H p a$ I to generate the OHFV- $\Delta \mathrm{NS} 1$ clone. To generate OHFV- $\triangle$ NS1-Gluc, the Gluc2A sequence was introduced by overlap PCR to replace the deleted NS1 sequence. Initially, the Gluc2A sequence was amplified using the plasmid pACYC-WNV-Gluc- $\Delta \mathrm{NS} 1$ as a template (Zhang et al. 2017a). The fragment encompassing the C-terminal 54 aa of the NS1 gene and NS2A was amplified using $\mathrm{OHF}-\mathrm{IC}$ as a template. The two fragments were fused by overlapping PCR and inserted into OHFV- $\Delta \mathrm{NS} 1$ between the restriction sites $\mathrm{Cla}$ I and Hpa I.

pBABEpuro-OHFV-NS1 was constructed by standard PCR using OHF-IC as a template with the BamH I and EcoR I restriction sites. The NS1 cassette contains fulllength NS1 with an N-terminal signal peptide sequence (the last 24 aa of the envelope protein) and C-terminal HAtag. All clones were sequenced prior to subsequent experiments.

\section{Western Blotting Assay}

Total proteins from FuGENE ${ }^{\circledR}$ HD (Promega, Madison, WI, USA) transfected BHK21 cells at $36 \mathrm{~h}$ post-transfection were lysed by RIPA buffer (Beyotime Biotechnology, Shanghai, China) and separated by sodium dodecyl sulfate-polyacrylamide gel electrophoresis (12\%). Proteins were then transferred onto $0.2-\mu \mathrm{m}$ polyvinylidene fluoride membranes (Bio-Rad, Hercules, CA, USA), followed by blocking with 5\% skim milk (Bio-Rad) in $1 \times$ TBST and sequential incubation with anti-HA (1:1000) and secondary anti-mouse $\mathrm{IgG}$ conjugated to horseradish peroxidase (1:2000, Beyotime Biotechnology). After three washes with $1 \times$ TBST, the signals were detected with a chemiluminescence system (ChemiDoc, Bio-Rad).

\section{In Vitro RNA Transcription and Transfection}

The OHFV- $\Delta$ NS1 and OHFV- $\Delta$ NS1-Gluc cDNA plasmids were linearized by $X b a$ I and purified by phenol/chloroform extraction. The linearized cDNA was then subjected to in vitro transcription with the mMESSAGE mMACHINE ${ }^{\circledR}$ T7 Kit (Ambion, Foster City, CA, USA) according to the manufacturer's protocols. The resulting RNA was dissolved in nuclease-free water and quantified by NanoDrop spectrophotometry (Thermo Fisher Scientific, Waltham, MA, USA). Next, $1 \mu \mathrm{g}$ RNA was transfected into BHK21 or $\mathrm{BHK} 21_{\mathrm{NS} 1}$ cells with reagent DMRIE-C (Invitrogen, Carlsbad, CA, USA). At different time points after transfection, the supernatants were collected. All experiments involving deficient virus rescue and passaging were performed under BSL3 conditions.

\section{Establishment of a Stable BHK21 $1_{\mathrm{NS} 1}$ Cell Line Expressing OHFV-NS1}

The retroviral vector system was used to generate a stable BHK21 cell line expressing NS1. Briefly, the constructs pBABEpuro-OHFV-NS1, m57, and VSV-G were cotransfected into $293 \mathrm{~T}$ cells by using calcium phosphate-DNA precipitates. At $6 \mathrm{~h}$ post-transfection (hpt), half of the culture medium was replaced with an equal volume of fresh medium. The combined retroviral supernatants were harvested at 48 and $72 \mathrm{hpt}$ followed by filtration through a $0.45-\mu \mathrm{m}$ filter, and then used to infect naïve BHK21 cells in the presence of $8 \mu \mathrm{g} / \mathrm{mL}$ polybrene. At $2 \mathrm{~h}$ post-infection, the medium was removed and replaced with fresh culture medium. On the second day, the cells were selected with medium containing $0.8 \mu \mathrm{g} / \mathrm{mL}$ of puromycin. After several rounds of puromycin selection, monoclonal cells were picked and amplified.

\section{IFA}

BHK21 and BHK21 $1_{\mathrm{NS} 1}$ cells cultured on cover slips were fixed in cold $\left(-20{ }^{\circ} \mathrm{C}\right) 5 \%$ acetone in methanol at $25^{\circ} \mathrm{C}$ for $10 \mathrm{~min}$. After washing three times with PBS (pH 7.4), the fixed cells were sequentially incubated with anti-HA tag or OHFV-NS3 antibody (1:200 dilution in PBS) and goat-anti-mouse IgG conjugated with fluorescein isothiocyanate (1:125 dilution in PBS) for $1 \mathrm{~h}$. The slides were mounted with $95 \%$ glycerol and examined under a fluorescence microscope at $100 \times$ or $400 \times$ magnification. 


\section{Reverse Transcription Polymerase Chain Reaction (RT-PCR)}

Total RNA was extracted using Trizol reagent (Takara, Shiga, Japan) from BHK21 and BHK21 $1_{\mathrm{NS} 1}$ cells infected with OHFV- $\triangle \mathrm{NS} 1$ or OHFV- $\triangle \mathrm{NS} 1-G l u c$ virus. The obtained RNA was identified by RT-PCR using the PrimeScript One Step RT-PCR Kit (Takara) with primers OHFV-1964-F (5'-CATTCTCTGGGACAAAGCCGT-3') and OHFV-3822-R (5'-TGTTAGACTTCTGCGCAGCAC$\left.3^{\prime}\right)$, which spanned the E and NS2A regions.

\section{Growth Kinetics and Titers Determination of Complemented Virus}

The growth kinetics of complemented virus were evaluated in the BHK21 $1_{\mathrm{NS} 1}$ cell line (12-well plate, $1 \times 10^{5}$ cells/ well) and infected at a multiplicity of infection (MOI) of $0.05 \mathrm{IU} / \mathrm{cell}$ (infectious unit per cell). At the indicated time points post-infection, the culture supernatants were collected and used for viral titer detection. To determine the virus titer, $1 \times 10^{5}$ BHK21 $1_{\mathrm{NS} 1}$ cells were seeded into a 24-well plate with coverslips. The cells were infected with the virus of different dilutions for $1 \mathrm{~h}$ at $37{ }^{\circ} \mathrm{C}$ and covered with $2 \%$ methyl cellulose for culturing for 1 day. Infected cells were washed three times with PBS, fixed with 5\% acetone, and then subjected to IFA to count the number of NS3-positive foci.

\section{Luciferase Assay}

BHK $21_{\mathrm{NS} 1}$ cells infected by OHFV- $\Delta \mathrm{NS} 1-$ Gluc were lysed with $1 \times$ lysis buffer (Thermo Fisher Scientific). The luciferase activity of the cell extracts was assayed by mixing $20 \mu \mathrm{L}$ lysate with $50 \mu \mathrm{L}$ substrate, followed by incubation for $10 \mathrm{~min}$ for signal stabilization and detection of the light output using a Multimode Microplate Reader (Varioskan Flash, Thermo Fisher Scientific).

\section{Antiviral Treatment for Complemented Virus with Gluc Reporter Gene}

BHK21 $1_{\mathrm{NS} 1}$ cells were seeded into 12 -well plates at a density of $1 \times 10^{5}$ cells/well. Next, the cells were infected with OHFV- $\Delta$ NS1-Gluc virus at an MOI of $0.1 \mathrm{IU} /$ cell and cultured in medium containing different concentrations of NITD008. After $48 \mathrm{~h}$ of treatment, the cell lysates were obtained, and luciferase activity was measured as described above. The $50 \%$ effective concentration $\left(\mathrm{EC}_{50}\right)$ was calculated with GraphPad Prism software 5.0 (GraphPad, Inc., La Jolla, CA, USA).

\section{HTS Assays}

For HTS assays, 5000,7500 , or 10,000 BHK $21_{\text {NS1 } 1}$ cells were plated into 96-well plates at the indicated densities per well and incubated for $24 \mathrm{~h}$. Next, the cells were infected with OHFV- $\Delta$ NS1-Gluc virus at an MOI of $0.1 \mathrm{IU} / \mathrm{cell}$ and cultured in the presence of dimethyl sulfoxide or $3 \mu \mathrm{mol} / \mathrm{L}$ of NITD008. At $24 \mathrm{~h}$ post-infection (hpi), the infected cells were lysed, and luciferase activity was measured. All luciferase assays were performed with 6 replicates.

\section{Results}

\section{Complementation of Replication-defective OHFV- ANS1 with Entire NS1 in Trans}

Based on the results of efficient trans complementation of NS1 protein on YFV and WNV RNA with a large in-frame deletion in the NS1 gene generating replication defective viruses (Lindenbach and Rice 1997; Khromykh et al. 2000; Zhang et al. 2017a), we applied a similar strategy for establishing a replication-defective virus system of OHFV. A cDNA clone of OHFV- $\Delta$ NS1 with deletion of the 4-298 aa in the $N S 1$ gene (Fig. 1A) was first prepared in a background of the full-length cDNA clone of the OHFV strain Guriev (OHF-IC), which was constructed by inserting the $\mathrm{T} 7$ promoter as well as $\mathrm{T} 7$ terminator and $\mathrm{HDVr}$ sequence at the $5^{\prime}$ and $3^{\prime}$ ends of the genome, respectively (Yoshii et al. 2011). To efficiently express OHFV NS1 protein, we used a retroviral vector system to construct the pBABEpuro-OHFV-NS1 expression plasmid containing the last 24 aa of $\mathrm{E}$ at the $\mathrm{N}$-terminal to ensure the proper localization of NS1 and the presence of an HA-tag at the C-terminal for convenient detection of expression (Fig. 1B).

To determine the expression of NS1, BHK21 cells transfected with pBABEpuro-OHFV-NS1 plasmid were analyzed by Western blotting at $36 \mathrm{hpt}$. Because of the $\mathrm{N}$ linked glycosylation of NS1, two forms of NS1 (approximately $39 \mathrm{kDa}$ and $47 \mathrm{kDa}$ ) were observed (Blitvich et al. 1999) (Fig. 1C). To assess trans-complementation, NS1 expression plasmids and in vitro-transcribed recombinant OHFV- $\triangle$ NS1 RNA were sequentially transfected into BHK21 cells and an equal amount of OHFV- $\triangle$ NS1 RNA was transfected into BHK21 cells. IFA for complementation of NS1 for OHFV- $\triangle$ NS1 using an anti-OHFV-NS3 antibody revealed no IFA-positive cells in cells transfected with OHFV- $\triangle$ NS1 RNA alone (Fig. 1D), demonstrating that deletion of the NS1 gene abrogated RNA replication. In contrast, IFA-positive cells were observed for cells 
A

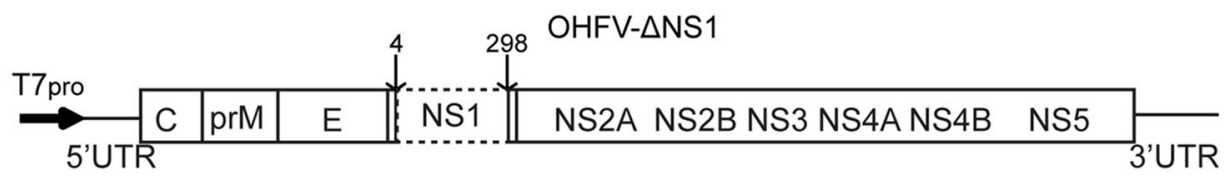

B

pBABEpuro-OHFV-NS1

\begin{tabular}{|c|c|c|}
\hline GGATCC ACC ATG & OHFV-E(24aa)-NS1+HA tag & TAA GAATTC \\
\hline $\mathrm{BamHI}$ & & EcoRI \\
\hline
\end{tabular}

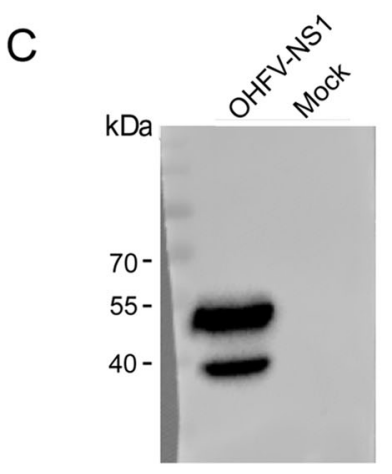

D
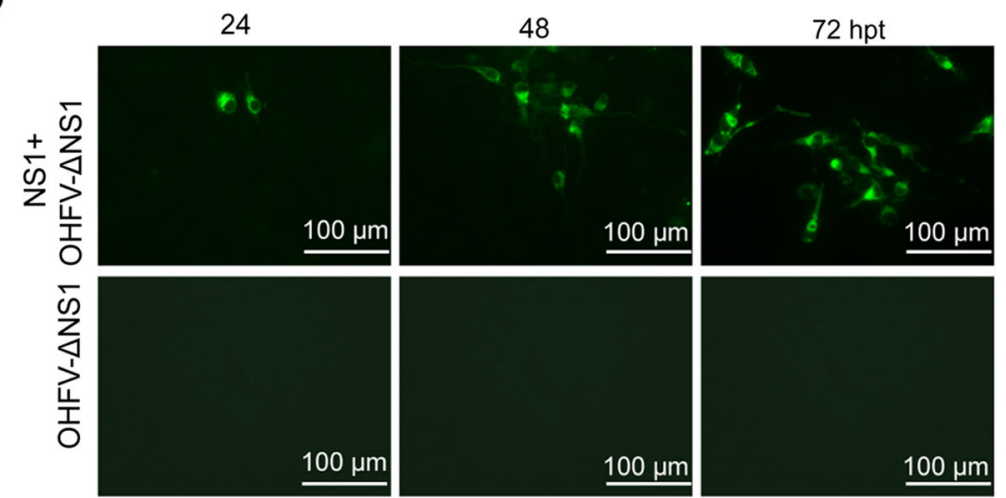

Fig. 1 Trans complementation of OHFV- $\Delta$ NS1 RNA with exogenous expression of NS1. A Schematic of the construction of OHFV- $\triangle$ NS1 clone. By fusion PCR, aa 4-298 in the NS1 gene were deleted using the full-length infectious clone as a template. Deletion sites are represented by a dotted box. B Schematic of the expression plasmid of OHFV-NS1. pBABEpuro retroviral vector and BamH I/EcoR I sites were used for clone construction. The cassette includes full-length NS1 with N-terminal 24 aa of E sequence and C-terminal HA-tag.

which co-transfected with the OHFV-NS1 expression plasmid and OHFV- $\triangle \mathrm{NS} 1 \mathrm{RNA}$, demonstrating that the replication defects of OHFV- $\triangle \mathrm{NS} 1$ can be trans-complemented efficiently by exogenous expression of NS1. Additionally, the number of positive cells increased from 24 to $72 \mathrm{hpt}$, indicating that OHFV- $\Delta \mathrm{NS} 1 \mathrm{RNA}$ can be packaged into a defective virus and propagated in the cells expressing homologous NS1 protein.

\section{Generation and Characterization of a Stable BHK21 Cell Line Expressing OHFV-NS1}

To improve the trans-complementation efficiency and constantly obtain defective virus of high yield, we attempted to establish a stable BHK21 cell line that could continuously and stably express NS1 using the retrovirus gene transfer system (Morgenstern and Land 1990; Zhang et al. 2017a) (Fig. 2A). Twenty-five resistant monoclonal cell clusters were selected and expanded. Seven cell lines (labeled as \#4, \#5, \#7, \#8, \#12, \#21, and \#24) from total 25
C Detection of expression of OHFV-NS1 by Western blotting assay using anti-HA antibody. D Trans complementation of OHFV- $\Delta$ NS1 with exogenous expression of NS1. BHK21 cells were sequentially transfected with $2 \mu \mathrm{g}$ pBABEpuro-OHFV-NS1 plasmid and $1 \mu \mathrm{g}$ OHFV- $\triangle$ NS1 RNA, and NS3 expression was analyzed by IFA at different times post-transfection. As a negative control, $1 \mu \mathrm{g}$ OHFV$\Delta$ NS1 RNA was transfected into BHK21 cells.

selected cell lines showed 100\% IFA-positive cells using the anti-HA antibody (Fig. 2B). To test the efficiency of trans complementation, OHFV- $\triangle \mathrm{NS} 1 \mathrm{RNA}$ was transfected into these seven cell lines and viral protein expression was visualized by IFA. Three cell lines (\#8, \#12, and \#24) showed high complementary efficiency, as increasing numbers of IFA-positive cells were observed from 24 to 48 hpt (Fig. 2C). Cell line \#8 was selected arbitrarily for subsequent analysis and designated as BHK21 $1_{\mathrm{NS} 1}$.

\section{Characterization of Trans-complemented Replication-defective OHFV- $\Delta$ NS1 Virus}

To confirm that defective OHFV- $\Delta$ NS1 virus was propagated only within the BHK21 $1_{\mathrm{NS} 1}$ cell line, supernatants from OHFV- $\Delta$ NS1 RNA-transfected BHK21 $1_{\mathrm{NS} 1}$ cells were used to infect naïve BHK21 cells and BHK21 $1_{\mathrm{NS} 1}$ cells. As expected, IFA-positive cells were only detected in BHK21 $1_{\mathrm{NS} 1}$ but not in BHK21 cells, suggesting that complemented virus was produced constantly by 
A

VSV-G, m57, pBABEpuro-OHFV-NS1 cotransfeceted in 293T cells

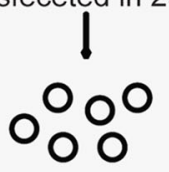

Supernantants acquiaition and filtration and infect BHK21 cells



Change the medium containing puromycin every 3 or 4 days

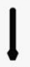

After a few rounds of puromycin selection, pick the monoclonal cells and expand culture

\section{B}

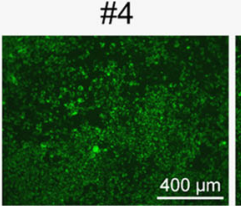

\#12

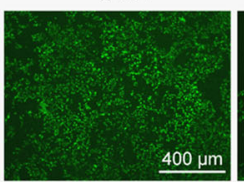

C
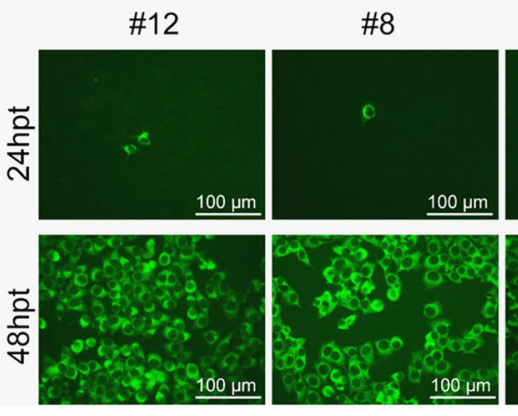

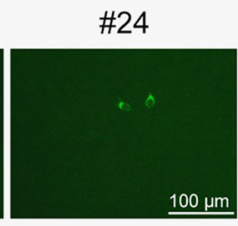

\#7

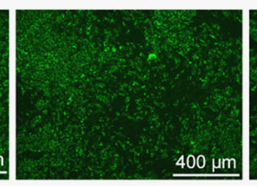

\#24
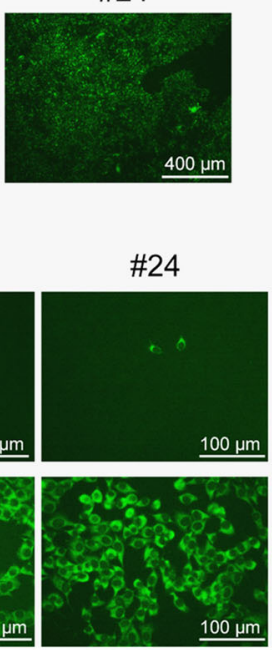

Fig. 2 Generation and characterization of BHK21 cell line stably expressing OHFV-NS1. A Flowchart for cell line selection. VSV-G, $\mathrm{m} 57$, and pBABEpuro-OHFV-NS1 plasmids were co-transfected into 293 T cells, and obtained retroviral supernatants were filtered with a $0.45-\mu \mathrm{m}$ filter. BHK21 cells were infected with recombinant retroviral virus containing polybrene. After $24 \mathrm{~h}$, cells were cultured in a medium containing $8 \mu \mathrm{g} / \mathrm{mL}$ puromycin. After several rounds of

complementation with NS1 in BHK21 $1_{\mathrm{NS} 1}$ cells and replication of OHFV- $\triangle \mathrm{NS} 1$ virus was confined to BHK21 $1_{\mathrm{NS} 1}$ cells. BHK21 $1_{\mathrm{NS} 1}$ cells were infected and the growth properties of OHFV- $\triangle \mathrm{NS} 1$ virus were analyzed over time. The virus titer of OHFV- $\Delta \mathrm{NS} 1$ reached $10^{7} \mathrm{IU} / \mathrm{mL}$ at 72 hpi (Fig. 3A), indicating that the complemented OHFV$\triangle \mathrm{NS} 1$ defective virus replicated efficiently in BHK21 $1_{\mathrm{NS} 1}$ cells.

To test the stability and biosafety of this complemented virus, the supernatants collected from OHFV- $\triangle$ NS1 RNA transfected $\mathrm{BHK} 21_{\mathrm{NS} 1}$ were designated as $\mathrm{P} 0$ and subjected to continuous passaging in BHK21 $1_{\mathrm{NS} 1}$ cells. Viral RNA from P1-P5 was subjected to RT-PCR with primers spanning the $\mathrm{E}$ and NS2A region. An approximately $1-\mathrm{kb}$ product from P1 to P5 was observed, which was smaller than that obtained using wild-type cDNA as a template (Fig. 3B). The product of P5 virus was sequenced without reversion, suggesting that deletion of NS1 was stably maintained and no reversion occurred. Additionally, supernatants from P1, P3 and P5 were used to infect fresh BHK $21_{\mathrm{NS} 1}$ and BHK21 cells. Comparable IFA-positive cells were only observed in BHK21 $1_{\mathrm{NS} 1}$, but not in BHK21 cells (Fig. 3C), conforming that the complemented replication-defective OHFV- $\Delta$ NS1 virus did not revert to the selection, visible monoclonal cells were picked and propagated. B Selected cell clones were subjected to IFA to determine the expression levels of NS1 using anti-HA antibody. The figure shows 7 cell lines that were $100 \%$ IFA positive. C Generation of OHFV- $\Delta$ NS1 virus in BHK21 $1_{\mathrm{NS} 1}$ cells. Obtained cell lines were transfected with $1 \mu \mathrm{g}$ recombinant OHFV- $\Delta$ NS1 RNA. Transfected cells were subjected to IFA at 24 and $48 \mathrm{hpt}$ using anti-NS3 antibody.

virulent wild-type virus for at least 5 passages. These results demonstrate the stability and safety of defective OHFV- $\Delta$ NS1 virus.

\section{Generation and Characterization of Complemented Replication-defective OHFV- $\Delta$ NS1-Gluc Reporter Virus}

To establish an OHFV high-throughput screening platform, we constructed OHFV- $\Delta$ NS1-Gluc by inserting the Gaussia luciferase (Gluc) reporter gene to replace NS1 (Fig. 4A). IFA-positive BHK21 $1_{\mathrm{NS} 1}$ cells transfected with OHFV- $\triangle$ NS1-Gluc RNA increased from 24 to $72 \mathrm{hpt}$, indicating that OHFV- $\Delta$ NS1-Gluc RNA was trans complemented by NS1 and the defective OHFV- $\Delta$ NS1-Gluc virus was produced efficiently (Fig. 4B). To compare the growth kinetics between complemented OHFV- $\triangle \mathrm{NS} 1$ and OHFV- $\Delta$ NS1-Gluc virus, BHK21 $1_{\mathrm{NS} 1}$ cells were infected with the two viruses. As shown in Fig. $4 \mathrm{C}$, the titer of OHFV- $\triangle$ NS1-Gluc was tenfold lower than that of OHFV$\Delta \mathrm{NS} 1$ before $60 \mathrm{hpi}$, with no significant replication difference after $72 \mathrm{hpi}$, demonstrating that the Gluc insertion had a subtle effect on the complementation efficiency of the defective virus. We then determined whether the Gluc 
A



C
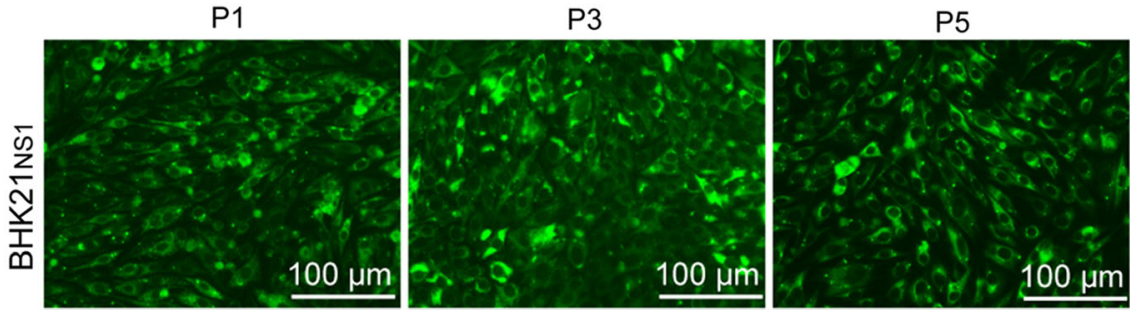

B
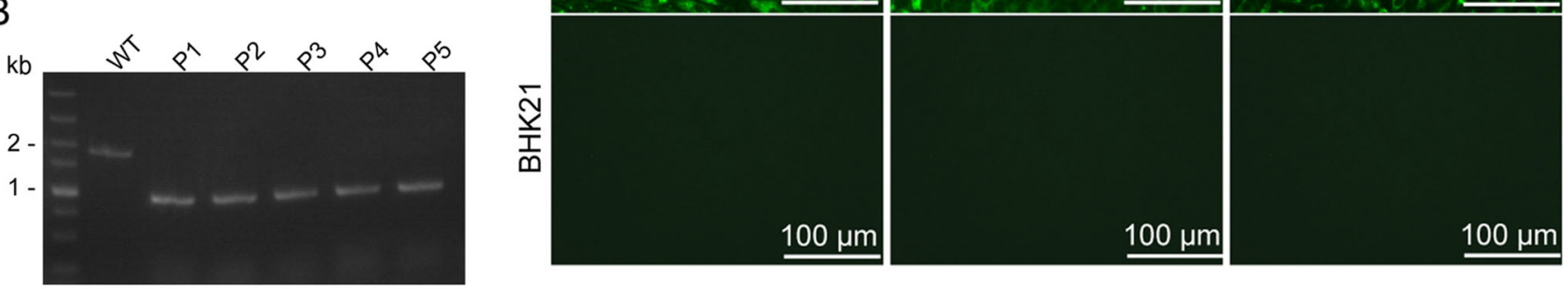

Fig. 3 Characterization of complemented-defective OHFV- $\Delta \mathrm{NS} 1$ virus. A Growth kinetics of OHFV- $\Delta \mathrm{NS} 1$ virus in $\mathrm{BHK} 21_{\mathrm{NS} 1}$ cells. B Genetic stability of OHFV- $\Delta$ NS1 virus. Total RNA was extracted from $\mathrm{BHK} 21_{\mathrm{NS} 1}$ cells infected with P1-P5 viruses. RT-PCR was performed with primer pairs spanning E and NS2A. As a control, WT

signal was correlated with viral replication within the OHFV- $\triangle \mathrm{NS} 1-\mathrm{Gluc}$ virus-infected $\mathrm{BHK}_{\mathrm{NS} 1}$ cell line. As shown in Fig. 4D, the luciferase signal was increased in proportion with the virus titer in culture. Additionally, the Gluc signal from $\mathrm{BHK} 21_{\mathrm{NS} 1}$ cells infected with different MOIs of viruses was elevated with an increasing viral load (Fig. 4E). Thus, the defective OHFV- $\Delta$ NS1-Gluc reporter virus was replication-competent and propagated efficiently within $\mathrm{BHK} 21_{\mathrm{NS} 1}$ cells, and replication of the OHFV$\Delta \mathrm{NS} 1-\mathrm{Gluc}$ reporter virus was surrogated by Gluc signals.

We also tested the stability of the OHFV- $\Delta$ NS1-Gluc reporter virus. P0 supernatants were blind-passaged in BHK21 $1_{\mathrm{NS} 1}$ cells. Continuous strong luciferase activities were observed in $\mathrm{BHK} 21_{\mathrm{NS} 1}$ cells infected with different passaging viruses, indicating that the reporter gene was stably maintained (Fig. 5A). To further confirm the stability of Gluc, total RNA was extracted for RT-PCR. The expected $1.5-\mathrm{kb}$ products of OHFV- $\mathrm{NNS1-Gluc}$ viruses were observed, which were smaller than those of the wildtype $(1.8-\mathrm{kb})$ and larger than those of OHFV- $\Delta \mathrm{NS} 1(1-\mathrm{kb})$ (Fig. 5B). Sequencing of P5 without NS1 reversion and Gluc deletion suggested that the OHFV- $\Delta$ NS1-Gluc reporter virus is stable for at least 5 passages.

\section{Antiviral Assay of Defective OHFV-DNS1-Gluc Reporter Virus}

Reporter viruses are powerful tools for rapid antiviral compound screening (Shang et al. 2013; Xu et al. 2015; Li et al. 2017; Zhang et al. 2017a). To validate whether this represents the products obtained by PCR using OHF-IC as a template. C Stability and biosafety of defective OHFV- $\triangle$ NS1 virus. BHK21 $1_{\mathrm{NS} 1}$ and BHK21 cells were infected with equal volumes of P1, P3, and P5 OHFV- $\triangle$ NS1 virus, followed by IFA analysis.

defective OHFV- $\Delta$ NS1-Gluc reporter virus can be used for antiviral assays, we used NITD008 to assess the performance of OHFV- $\Delta$ NS1-Gluc reporter virus in antiviral screening. NITD008, a mosquito-borne flavivirus antiviral (Yin et al. 2009), suppressed the replication of tick-borne flavivirus such as OHFV in vitro (Lo et al. 2016). With antiviral assay, Gluc activities were decreased in an NITD008 concentration-dependent manner (Fig. 6A). The $\mathrm{EC}_{50}$ was $0.15 \mu \mathrm{mol} / \mathrm{L}$, which was similar to the value of the cell-based flavivirus immunodetection $\mathrm{EC}_{50}$ (Lo et al. 2016).

The defective virus-based HTS assay was conducted in a 96-well plate format using NITD008 as a positive control drug using different numbers of $\mathrm{BHK} 21_{\mathrm{NS} 1}$ cells in each well. After $24 \mathrm{~h}$, the cells were incubated in medium containing virus and NITD008 or DMSO. As shown in Fig. $6 \mathrm{~B}$, the $\mathrm{Z}^{\prime}$ values were $>0.5$ in wells of seeded with 7500 and 10,000 cells, suggesting that the HTS assay was reliable under these two conditions for detecting the inhibitory effect of NITD008. For 10,000 cells/well and $24 \mathrm{~h}$ incubation, we confirmed that the replication-defective OHFV- $\Delta$ NS1-Gluc reporter virus can be used for HTS assays in a 96-well plate format to screen for inhibitors of OHFV.

\section{Discussion}

$\mathrm{OHFV}$ is classified as a BSL4 virus; the requirement for BSL4 containment facilities has prevented progress in OHFV research. Thus, an alternative to the authentic virus 
A

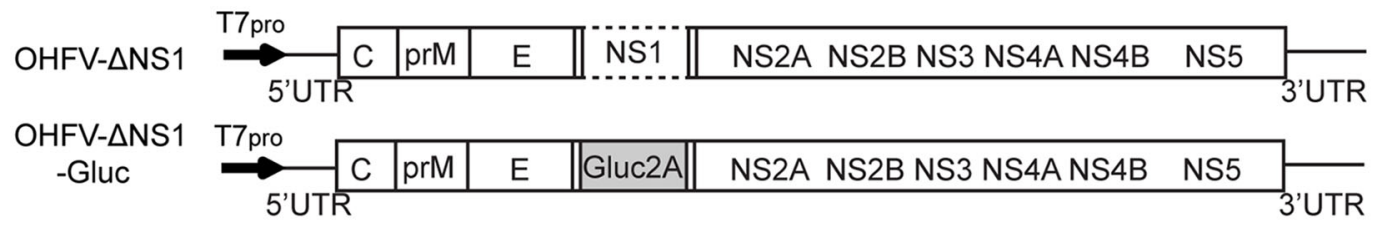

B

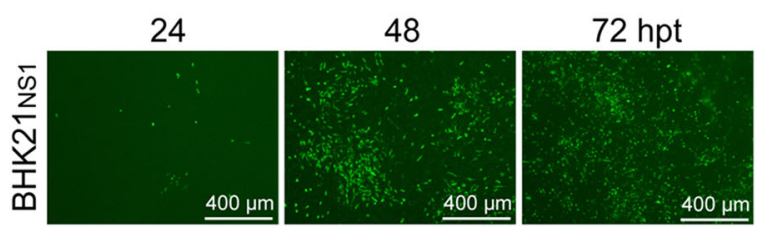

C

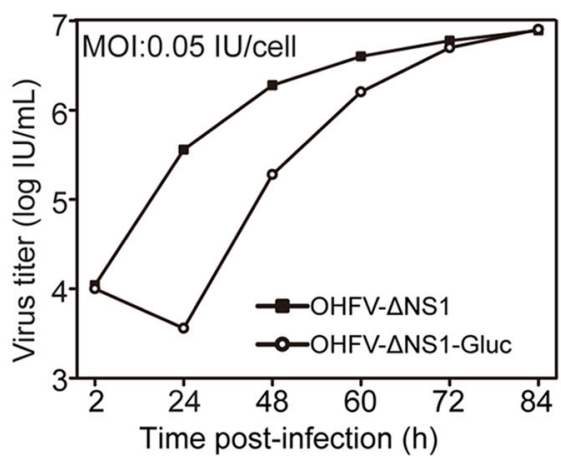

Fig. 4 Generation and characterization of complemented-defective OHFV- $\triangle$ NS1-Gluc virus. A Schematic of construction of OHFV$\Delta$ NS1-Gluc clone. The Gluc2A sequences were inserted to replace the NS1 gene. B Generation of complemented OHFV- $\triangle$ NS1-Gluc virus. OHFV- $\Delta$ NS1-Gluc RNA was transfected into $\mathrm{BHK} 21_{\mathrm{NS} 1}$ cells and NS3 expression was analyzed by IFA at different times posttransfection. C Comparison of growth kinetics between complemented OHFV- $\Delta \mathrm{NS} 1$ and OHFV- $\Delta \mathrm{NS} 1-\mathrm{Gluc}$ virus in $\mathrm{BHK} 21_{\mathrm{NS} 1}$ cells. BHK21 $1_{\mathrm{NS} 1}$ cells were infected at MOI of $0.05 \mathrm{IU} /$ cell. Supernatants at indicated times were harvested and titers were determined as described in "Materials and Methods" section. D Correlation between viral titer and Gluc signals of defective OHFV$\Delta$ NS1-Gluc virus in BHK21 $1_{\mathrm{NS} 1}$ cells. BHK21 $1_{\mathrm{NS} 1}$ cells were infected

is needed. In this study, we generated safe and stable replication-defective OHFV- $\triangle \mathrm{NS} 1$ and OHFV$\Delta$ NS1-Gluc viruses for studying virus replication and antiviral screening in BSL2 laboratories.

Replicons, the VLP system, and defective viruses are powerful tools for studying viral replication, antiviral screening, and vaccine candidate development for many flaviviruses (Lo et al. 2003; Hayasaka et al. 2004; Qing et al. 2010; Zhang et al. 2017a, c; Li et al. 2018; Salat et al. 2018). Because they lack capsid genes, YFV- and WNVdefective viruses have been generated as vaccine candidates in trans-complementing cell lines expressing high levels of the capsid or all three viral structural proteins (Mason et al. 2006). Moreover, replication-defective virus

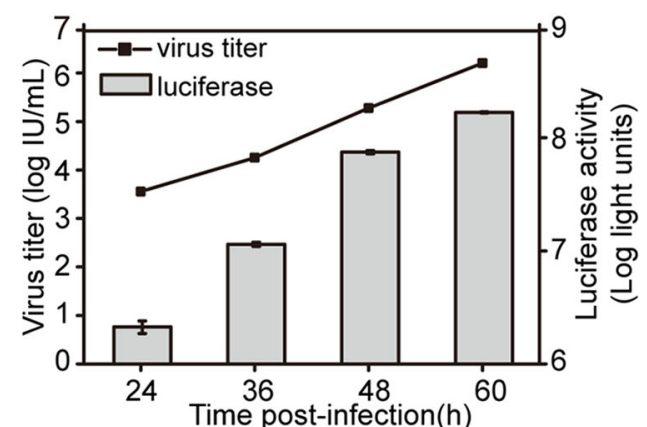

$E$

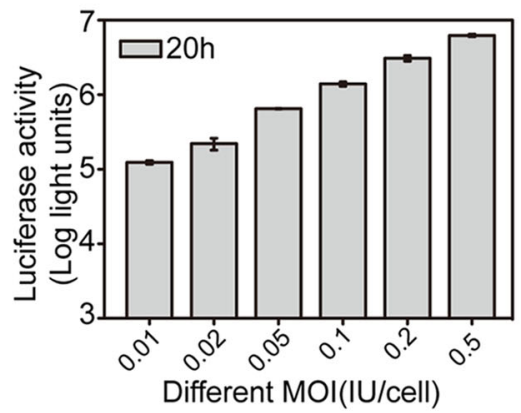

with OHFV- $\triangle \mathrm{NS} 1-\mathrm{Gluc}$ virus at MOI of $0.05 \mathrm{IU} /$ cell in a 12 -well format. Supernatants and cell lysates were collected at indicated times. The titer of supernatants and Gluc signals of cell lysates were determined as described in "Materials and Methods" section. The data shown are the mean values of triplicate measurements with error bars showing standard deviation. E Correlation between Gluc activity and viral load of OHFV- $\Delta \mathrm{NS} 1-\mathrm{Gluc}$ virus in $\mathrm{BHK} 21_{\mathrm{NS} 1}$ cells. $\mathrm{BHK} 21_{\mathrm{NS} 1}$ cells were infected with $\mathrm{OHFV}-\Delta \mathrm{NS} 1-\mathrm{Gluc}$ virus at MOI of $0.01,0.02,0.05,0.1,0.2$, and $0.5 \mathrm{IU} /$ cell in a 12 -well format. At 20 hpi, cell lysates were collected and analyzed for Gluc activity. The data shown are the mean values of triplicate measurements with error bars showing standard deviation.

genomic WNV RNA with a range of large deletions in NS1 was successfully trans-complemented using a replicon cell line or by foreign expression of NS1 (Khromykh et al. 2000; Zhang et al. 2017a). The defective viruses generated by these complementation strategies exhibit genetic stability and biosafety, making them useful for HTS of antiviral compounds in BSL2 laboratories.

Previous studies showed that for WNV virus, approximately $84 \%-97 \%$ large-frame deletions in the NS1 gene can be efficiently complemented by exogenous NS1 expression (Khromykh et al. 2000; Zhang et al. 2017a). Here, we constructed the cDNA clone of OHFV with deletion of the NSI gene (4-295 aa). The first three amino acids of NS1 were retained to ensure proper cleavage by 


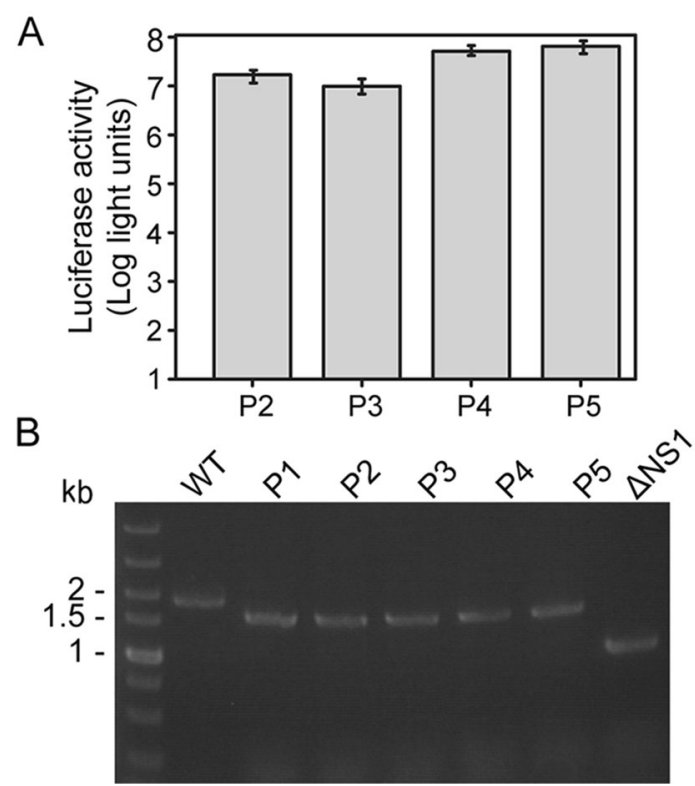

Fig. 5 Characterization of stability and safety of complementeddefective OHFV- $\triangle$ NS1-Gluc virus. A Gluc signals of OHFV- $\triangle$ NS1Gluc viruses during passaging. The OHFV- $\Delta$ NS1-Gluc virus was passaged for five rounds in BHK21 $1_{\mathrm{NS} 1}$ cells and cell lysates were obtained at 72 hpi. Data shown are the mean values of triplicate measurements with error bars showing standard deviation. B Genetic stability of Gluc reporter gene of passaged reporter virus. Total RNA was extracted from BHK21 $1_{\mathrm{NS} 1}$ cells infected with passaging OHFV$\triangle \mathrm{NS1-Gluc}$ virus and RT-PCR was performed. As a control, WT represents the products obtained by PCR using OHF-IC cDNA as a template and $\triangle \mathrm{NS} 1$ represents the products of RT-PCR with OHFV$\Delta \mathrm{NS} 1 \mathrm{RNA}$. The products size of WT, P1-P5, and $\Delta \mathrm{NS} 1$ were 1.8 , 1.5 , and $1 \mathrm{~kb}$, respectively.

cellular signal peptidase, and the presence of the C-terminus of NS1 was essential for efficient trans complementation (Khromykh et al. 2000). Through sequential transfection, recombinant OHFV- $\Delta$ NS1 RNA was transcomplemented efficiently by exogenous expression of NS1 protein (Fig. 1D). By generating a BHK21 $1_{\mathrm{NS} 1}$ cell line continuously expressing NS1, the yield of OHFV- $\triangle \mathrm{NS} 1$ virus was improved (Fig. 2). Additionally, this defective virus stably and efficiently replicated in $\mathrm{BHK} 21_{\mathrm{NS} 1}$ cells with no recombination within five rounds of passaging, indicating its potential as a vaccine candidate (Fig. 3). This complemented OHFV- $\Delta$ NS1 defective virus will be a powerful tool for studying the replication mechanism and pathogenesis of OHFV in BSL2 laboratories.

To achieve HTS for OHFV inhibitors, complemented OHFV- $\Delta$ NS1-Gluc virus with a Gluc reporter gene was obtained. Because of the plasticity of the NS1 gene which can highly tolerate the insertion of foreign genes (Eyre et al. 2017), we inserted Gluc2A sequences to replace NS1 (Fig. 4A), which differed from previous strategies of inserting a reporter gene between the $5^{\prime}$ untranslated region and structural gene (Shang et al. 2013; Xu et al. 2015;
A

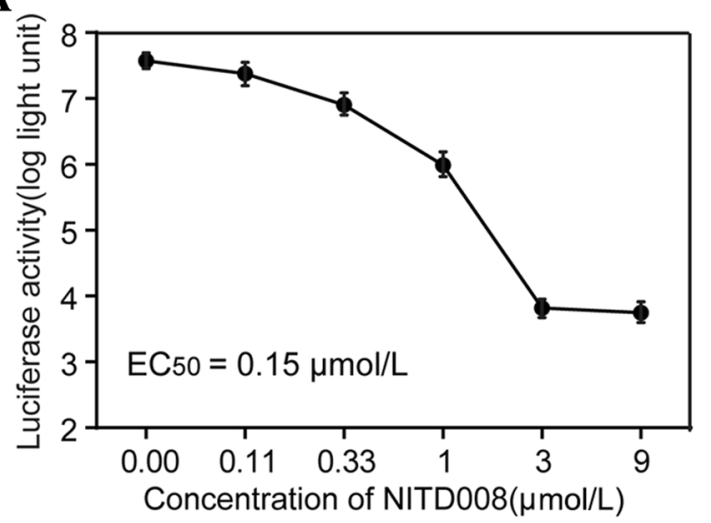

B

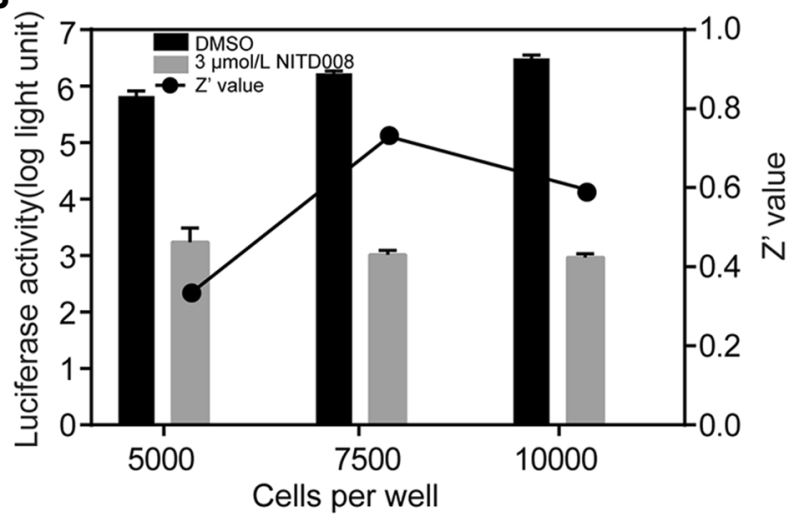

Fig. 6 Antiviral activity of NITD008 on OHFV- $\Delta$ NS1-Gluc virus in BHK $21_{\text {NS1 }}$ cells. A Antiviral activity of NITD008 on OHFV- $\Delta$ NS1Gluc virus replication. BHK21 $1_{\mathrm{NS} 1}$ cells were infected with OHFV$\Delta \mathrm{NS} 1-\mathrm{Gluc}$ virus and incubated with different concentrations of NITD008. After $48 \mathrm{~h}$, cells were lysed and Gluc activity was examined. Three independent experiments were performed in duplicate, with error bars indicating standard deviation. B Optimization of HTS assay using OHFV- $\Delta$ NS1-Gluc viruses. Different densities of BHK21 $1_{\mathrm{NS} 1}$ cells were seeded into a 96-well plate. Cells were infected with OHFV- $\triangle$ NS1-Gluc virus and incubated with a medium containing $3 \mu \mathrm{mol} / \mathrm{L}$ NITD008. At $24 \mathrm{hpi}$, the cells were lysed and assayed for luciferase activity. The $Z^{\prime}$ value was calculated to evaluate the effectiveness of the HTS assay.

Zhang et al. 2017a). The OHFV- $\Delta$ NS1-Gluc virus showed comparable replication characteristics to OHFV- $\Delta \mathrm{NS} 1$ virus (Fig. 4C). By monitoring the Gluc activity of cells containing different virus loads, we found that replication of this defective virus was effectively reflected by Gluc signals (Fig. 4D, 4E). Additionally, this defective OHFV$\triangle$ NS1-Gluc virus produced steady and robust luciferase activity for 5 rounds of passaging in BHK21 $1_{\mathrm{NS} 1}$ cells (Fig. 5), displaying relatively good genetic stability.

The inhibition effect of NITD008 on OHFV- $\Delta$ NS1-Gluc replication was concentration-dependent (Fig. 6A). In a 96-well format, we optimized the parameters of the HTS assay and found that the $Z^{\prime}$ value was between 0.5 and 1 , 
revealing that this complemented reporter virus can be used for HTS screening of OHFV inhibitors (Fig. 6B).

In conclusion, we constructed stable, safe defective OHFV- $\triangle \mathrm{NS} 1$ and OHFV- $\Delta \mathrm{NS} 1-\mathrm{Gluc}$ viruses that can be handled under BSL 2 conditions for at least 5 passages. This novel complemented replication-defective system is a powerful tool for researching virus replication, pathogenesis, and HTS of antiviral compounds for OHFV.

Acknowledgements This work was supported by National Science and Technology Major Project on Important Infectious Diseases Prevention and Control (2018ZX10734404-010) and National Key Research and Development Program of China (2018YFA0507201). We thank the Center for Instrumental Analysis and Metrology, Wuhan Institute of Virology, Wuhan National Biosafety Level 4 Lab of CAS and Wuhan Key Laboratory of Special Pathogens and Biosafety for helpful assistance during the course of the work.

Author Contributions BZ designed the experiments. QZ, NL, CD, and $\mathrm{ZZ}$ carried out the experiments. HY, and BZ analyzed the data. $\mathrm{QZ}, \mathrm{XL}, \mathrm{CD}$, and BZ wrote the paper. All authors read and approved the final manuscript.

\section{Compliance with Ethical Standards}

Conflict of interest The authors declare that they have no conflict of interest.

Animal and Human Rights Statement This article does not contain any studies with human or animal subjects performed by any of the authors.

\section{References}

Blitvich BJ, Scanlon D, Shiell BJ, Mackenzie JS, Hall RA (1999) Identification and analysis of truncated and elongated species of the flavivirus NS1 protein. Virus Res 60:67-79

Charrel RN, Attoui H, Butenko AM, Clegg JC, Deubel V, Frolova TV, Gould EA, Gritsun TS, Heinz FX, Labuda M, Lashkevich VA, Loktev V, Lundkvist A, Lvov DV, Mandl CW, Niedrig M, Papa A, Petrov VS, Plyusnin A, Randolph S, Suss J, Zlobin VI, de Lamballerie X (2004) Tick-borne virus diseases of human interest in Europe. Clin Microbiol Infect 10:1040-1055

Dobler G (2010) Zoonotic tick-borne flaviviruses. Vet Microbiol 140:221-228

Eyre NS, Johnson SM, Eltahla AA, Aloi M, Aloia AL, McDevitt CA, Bull RA, Beard MR (2017) Genome-wide mutagenesis of dengue virus reveals plasticity of the NS1 protein and enables generation of infectious tagged reporter viruses. J Virol 91:e01455-17

Halfmann P, Kim JH, Ebihara H, Noda T, Neumann G, Feldmann H, Kawaoka Y (2008) Generation of biologically contained Ebola viruses. Proc Natl Acad Sci USA 105:1129-1133

Hayasaka D, Yoshii K, Ueki T, Iwasaki T, Takashima I (2004) Subgenomic replicons of Tick-borne encephalitis virus. Arch Virol 149:1245-1256

Khromykh AA, Sedlak PL, Westaway EG (2000) cis- and trans-acting elements in flavivirus RNA replication. J Virol 74:3253-3263

Lani R, Moghaddam E, Haghani A, Chang LY, AbuBakar S, Zandi K (2014) Tick-borne viruses: a review from the perspective of therapeutic approaches. Ticks Tick Borne Dis 5:457-465
Li XF, Li XD, Deng CL, Dong HL, Zhang QY, Ye Q, Ye HQ, Huang XY, Deng YQ, Zhang B, Qin CF (2017) Visualization of a neurotropic flavivirus infection in mouse reveals unique viscerotropism controlled by host type I interferon signaling. Theranostics 7:912-925

Li JQ, Deng CL, Gu D, Li X, Shi L, He J, Zhang QY, Zhang B, Ye HQ (2018) Development of a replicon cell line-based high throughput antiviral assay for screening inhibitors of Zika virus. Antivir Res 150:148-154

Lindenbach BD, Rice CM (1997) trans-Complementation of yellow fever virus NS1 reveals a role in early RNA replication. J Virol 71:9608-9617

Lo MK, Tilgner M, Bernard KA, Shi PY (2003) Functional analysis of mosquito-borne flavivirus conserved sequence elements within $3^{\prime}$ untranslated region of West Nile virus by use of a reporting replicon that differentiates between viral translation and RNA replication. J Virol 77:10004-10014

Lo MK, Shi PY, Chen YL, Flint M, Spiropoulou CF (2016) In vitro antiviral activity of adenosine analog NITD008 against tickborne flaviviruses. Antivir Res 130:46-49

Mason PW, Shustov AV, Frolov I (2006) Production and characterization of vaccines based on flaviviruses defective in replication. Virology 351:432-443

Morgenstern JP, Land H (1990) Advanced mammalian gene transfer: high titre retroviral vectors with multiple drug selection markers and a complementary helper-free packaging cell line. Nucleic Acids Res 18:3587-3596

Orlinger KK, Hofmeister Y, Fritz R, Holzer GW, Falkner FG, Unger B, Loew-Baselli A, Poellabauer EM, Ehrlich HJ, Barrett PN, Kreil TR (2011) A tick-borne encephalitis virus vaccine based on the European prototype strain induces broadly reactive crossneutralizing antibodies in humans. J Infect Dis 203:1556-1564

Pierson TC, Sanchez MD, Puffer BA, Ahmed AA, Geiss BJ, Valentine LE, Altamura LA, Diamond MS, Doms RW (2006) A rapid and quantitative assay for measuring antibody-mediated neutralization of West Nile virus infection. Virology 346:53-65

Pohjala L, Utt A, Varjak M, Lulla A, Merits A, Ahola T, Tammela P (2011) Inhibitors of alphavirus entry and replication identified with a stable Chikungunya replicon cell line and virus-based assays. PLoS ONE 6:e28923

Qing M, Liu W, Yuan Z, Gu F, Shi PY (2010) A high-throughput assay using dengue-1 virus-like particles for drug discovery. Antivir Res 86:163-171

Ruzek D, Yakimenko VV, Karan LS, Tkachev SE (2010) Omsk haemorrhagic fever. Lancet 376:2104-2113

Salat J, Formanova P, Hunady M, Eyer L, Palus M, Ruzek D (2018) Development and testing of a new tick-borne encephalitis virus vaccine candidate for veterinary use. Vaccine 36:7257-7261

Scholle F, Girard YA, Zhao Q, Higgs S, Mason PW (2004) transPackaged West Nile virus-like particles: infectious properties in vitro and in infected mosquito vectors. $\mathbf{J}$ Virol 78:11605-11614

Shang B, Deng C, Ye H, Xu W, Yuan Z, Shi PY, Zhang B (2013) Development and characterization of a stable eGFP enterovirus 71 for antiviral screening. Antivir Res 97:198-205

Shi PY, Tilgner M, Lo MK (2002) Construction and characterization of subgenomic replicons of New York strain of West Nile virus. Virology 296:219-233

Si L, Xu H, Zhou X, Zhang Z, Tian Z, Wang Y, Wu Y, Zhang B, Niu Z, Zhang C, Fu G, Xiao S, Xia Q, Zhang L, Zhou D (2016) Generation of influenza A viruses as live but replicationincompetent virus vaccines. Science 354:1170-1173

Wang SR, Zhang QY, Wang JQ, Ge XY, Song YY, Wang YF, Li XD, Fu BS, Xu GH, Shu B, Gong P, Zhang B, Tian T, Zhou X (2016) Chemical targeting of a G-quadruplex RNA in the Ebola virus L gene. Cell Chem Biol 23:1113-1122 
Xu LL, Shan C, Deng CL, Li XD, Shang BD, Ye HQ, Liu SQ, Yuan ZM, Wang QY, Shi PY, Zhang B (2015) Development of a stable Gaussia luciferase enterovirus 71 reporter virus. J Virol Methods 219:62-66

Yin Z, Chen YL, Schul W, Wang QY, Gu F, Duraiswamy J, Kondreddi RR, Niyomrattanakit P, Lakshminarayana SB, Goh A, Xu HY, Liu W, Liu B, Lim JY, Ng CY, Qing M, Lim CC, Yip A, Wang G, Chan WL, Tan HP, Lin K, Zhang B, Zou G, Bernard KA, Garrett C, Beltz K, Dong M, Weaver M, He H, Pichota A, Dartois V, Keller TH, Shi PY (2009) An adenosine nucleoside inhibitor of dengue virus. Proc Natl Acad Sci USA 106:20435-20439

Yoshii K, Holbrook MR (2009) Sub-genomic replicon and virus-like particles of Omsk hemorrhagic fever virus. Arch Virol 154:573-580

Yoshii K, Ikawa A, Chiba Y, Omori Y, Maeda J, Murata R, Kariwa H, Takashima I (2009) Establishment of a neutralization test involving reporter gene-expressing virus-like particles of tickborne encephalitis virus. J Virol Methods 161:173-176
Yoshii K, Igarashi M, Ito K, Kariwa H, Holbrook MR, Takashima I (2011) Construction of an infectious cDNA clone for Omsk hemorrhagic fever virus, and characterization of mutations in NS2A and NS5. Virus Res 155:61-68

Youn S, Li T, McCune BT, Edeling MA, Fremont DH, Cristea IM, Diamond MS (2012) Evidence for a genetic and physical interaction between nonstructural proteins NS1 and NS4B that modulates replication of West Nile virus. J Virol 86:7360-7371

Zhang HL, Ye HQ, Deng CL, Liu SQ, Shi PY, Qin CF, Yuan ZM, Zhang B (2017a) Generation and characterization of West Nile pseudo-infectious reporter virus for antiviral screening. Antivir Res 141:38-47

Zhang HL, Ye HQ, Liu SQ, Deng CL, Li XD, Shi PY, Zhang B (2017b) West Nile virus NS1 antagonizes interferon beta production by targeting RIG-I and MDA5. J Virol 91:e02396-16

Zhang QY, Li XD, Liu SQ, Deng CL, Zhang B, Ye HQ (2017c) Development of a stable Japanese encephalitis virus replicon cell line for antiviral screening. Arch Virol 162:3417-3423 